(c) 2010 IEEE. Personal use of this material is permitted. Permission from IEEE must be obtained for all other uses, in any current or future media, including reprinting/republishing this material for advertising or promotional purposes, creating new collective works, for resale or redistribution to servers or lists, or reuse of any copyrighted component of this work in other works. 


\title{
A Framework for Patient Practitioner Information Exchange
}

\author{
David Forbes, Amandeep Sidhu, Jaipal Singh \\ Digital Ecosystem and Business Intelligence Institute \\ Curtin University of Technology \\ Perth, Australia \\ david.e.forbes@postgrad.curtin.edu.au, \{a.sidhu, j.singh\}@curtin.edu.au
}

\begin{abstract}
The global Type 2 Diabetes Mellitus (T2DM) epidemic imposes a heavy burden on communities that are ethnically vulnerable to the disease and further disadvantaged by socio-economic circumstance and cultural communications barriers. Aboriginal communities in rural and remote Western Australia are representative of these high-risk groups. Indigenous patients needing continuous management of T2DM are also experiencing disproportionate risk of co-morbidities and hospitalizations compared with nonindigenous patients. Type 2 Diabetes Mellitus (T2DM) is often described as 'the lifestyle disease'. Within clinical care and patient quality of life management domains, T2DM presents both the healthcare practitioner and the patient with a mosaic of complexities. Information processing demands for self-management of diabetes are extensive, requiring constant self monitoring and assessment of the illness state in order to apply per instance and per condition the most appropriate form of control. In this work we introduce a primary care communications concept tool centered upon optimization of the Patient-Practitioner Interview Encounter (PPIE). The target beneficiary is the Aboriginal T2DM patient living in Western Australia. A vital part of our design effort is therefore dedicated to understanding and responding to the cultural domain barriers, challenges and opportunities of this specific health care environment.
\end{abstract}

\section{Introduction}

The health literacy (HL) of the T2DM patient is a component that may at its extremes either mitigate or exacerbate care complexity, influencing PPIE efficacy and the consequential effect on outcomes. The World Health
Organization (WHO) states that 'Health literacy represents the cognitive and social skills which determine the motivation and ability of individuals to gain access to, understand and use information in ways which promote and maintain good health' [1]. Ratzan and Parker describe health literacy as the degree to which individuals have the capacity to obtain, process, and understand basic health information and services needed to make appropriate health decisions [2].

Several inconclusive studies have examined the possibility of a relationship of cognition with health literacy, a factor to be taken into account when assessing the potential for introducing a new health care communications interface for the benefit of and use by, T2DM patients. Within the T2DM population there is no indisputable data to suggest that the disease alone directly causes or contributes to cognitive decline. How cognitive decline evolves remains uncertain [3], [4]. For the purposes of this research we assume that the majority of the patient pool for whom enhanced communications support is intended will not be suffering from impaired cognition.

Communications weaknesses and failings in diagnoses, treatment and care practices are contributing to continuing growth of co-morbidities, hospitalizations for complications and premature deaths in Western Australia (WA) Aboriginal communities [5]. The thinly spread ranks of over-stretched practitioners, particularly qualified nurses in remote areas, are constantly faced with mastering the complex challenges of socio-cultural engagement without significant advances in communications capabilities. Much has been published on the subject of patient-practitioner consultation and several models have been offered by physicians and other health professionals who are aware of the need to improve the outcome of their encounters with patients [6], [7]. The human body and mind together comprise a repository of knowledge. That repository and/or the extensive reach of its content are not automatically or 
predictably accessible and sharable. A number of barriers complicate and adversely influence communications affecting clinical diagnosis of the human patient. Australia's Aboriginal population in particular has struggled with the cultural gap that exists between the Indigenous view of the world and the dominant non-Indigenous culture that has overwhelmed that perspective and dictated the direction of social norms and healthcare. According to Trudgen this has led to harmful misunderstandings on such aspects of health as diet and nutrition [8].

TD2M patients with multiple barriers are generally less adherent to their care plans. For the patient who is already confronted with the incessant intricacies of self-management, health literacy weaknesses present the elevated risk of further diminution of opportunity to contribute to and to receive optimal care. In recognition of this, the importance of barrier and strategy identification has been highlighted [9]. Several studies have shown benefits from health literacy programs, some specifically in the diabetes patient population and in chronic disease care for older patient groups where functionality affecting health literacy is especially at risk [10], [11], [12].

The hidden PPIE knowledge that would potentially deliver a better diagnosis and health care outcome is difficult to qualify and quantify. We don't know what we don't know. We have two primary objectives aimed at strengthening the value of PPIEs:

1) We will employ emerging and new communications technologies to significantly enhance the knowledge capture and pragmatic contribution of practitionerpatient diagnostic interactions to ultimately optimize treatment and care.

2) With this approach we will ensure sufficient versatility and flexibility to accommodate heterogeneity of culture, intellect and reciprocal dialogue.

This concept mitigates the unwanted consequences of dominant influence and bias of clinical Standard English in the patient-practitioner conversational dialogue; but protects the clinical validity of the patient diagnosis, treatment and care plan. The communications interface will include education modules for the prevention of T2DM and associated complications, thereby widening the audience scope to extended families and personal carers.

\section{Background}

A legitimate question about a prototype project of this nature concerns whether the hardware and software technology tools for delivering the intended outcome will be accessible, acceptable to and usable by Aboriginal patients and their communities. Contemporary obstacles identified include weak communications infrastructure, e.g. broadband connectivity, lack of current computer access and usage by Indigenous people [13]. UNESCO world studies report that the most obvious challenge to Indigenous people using ICTs for intercultural dialogue is their inadequate access to technology [14].

A literature review in 2004 examined the hypothesis that low adoption of ICT by Indigenous Australians was influenced by Western values embodied in the technology. The researchers found instead that there was an 'overwhelmingly enthusiastic response towards computers' by school children, and capabilities limited only by costassociated technology access difficulties, isolation, poor telecommunications infrastructure and low computer skills [13].

A preliminary study of mobile phone adoption on a remote island in the Torres Strait disclosed the unexpected use by the Indigenous community of text messaging on mobile phones, as well as calls and text messages in the local language. The tentative conclusion was that ICT must go beyond cultural oral strengths, to match areas of motivation such as communication with family [15].

The Health Interactive Technology Network (HITNet) in Queensland develops and deploys creative media solutions to reduce Indigenous health inequalities. These media concepts favour the use of 'performative' and participative content in Indigenous communities (as opposed to narrative text) because they are more attuned to listening and watching versus literacy-based media [16].

We recognize that ICT is intrinsically a management tool, not a panacea for healthcare inadequacies. Xie et al. caution that computer-based communications exacerbates ambiguity and misunderstanding among parties with different cultural backgrounds [17]. Thoughtful design of applications and User Interfaces (UIs) is essential, more so when intended users have had limited exposure to ICT and potential self-management of chronic conditions using electronic Point of Care (PoCT) and communications devices. These devices increasingly represent intelligent machine participation in PPIE. We are learning that westernized assumptions about the value of speech and written text are unreliable; such limitations fail to optimize the conversational modalities available when engaging different cultures [18].

\section{Methodology}

\section{Miscommunication}

A valuable insight to miscommunication in the Australian Aboriginal healthcare dialogue context is illustrated by Cass et al. [19]. In 2001 in Darwin, NT, five clinical interactions involving diagnosis and chronic disease management of Aboriginal patients, spanning a period of five months, were conducted and videotaped, with the agreement of all participants. Each interaction 
was followed with in-depth interviews, of individual patients and health workers. The conclusion of this study was that miscommunication is pervasive, and that trained interpreters provide only a partial solution. A shared understanding of key concepts 'was rarely achieved' and 'miscommunication often went unrecognised'. The frequent phenomena of 'gratuitous concurrence' i.e. the patient answering a yes/no type question by offering a response that the patient thinks the health worker would prefer to hear, was very apparent from this study. One nurse remarked 'I never even considered that they might be saying "yo" (yes) when they are really saying "no". I never even thought of it.' [19].

\section{Aboriginal English Ontology}

Several studies have been dedicated to augmenting human dialogue using ICT [20]. A computer aided linguistic translation system alone will not achieve the desired harvest of knowledge from communications. The Aboriginal English Ontology is prepared by mapping to SAE and T2DM clinical guidelines. Figure 1 gives the upper class scope, extending into subclasses. These are expressive of the complexity at play within the cognitive processes and socio-cultural differences between Aboriginal AE speakers and non-Indigenous SAE speakers. Please refer to our paper [21] for more details.

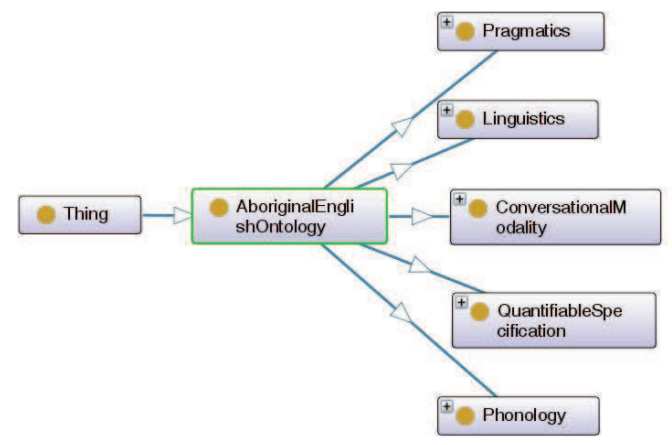

Fig. 1. Aboriginal English Ontology.

\section{Community Healthcare Ontology for T2DM}

Mehta [22] addresses the need for categorization of ontological resources into domain independent and domain specific components in order to augment conversational capabilities. As with translational based ontology discussions, the cautionary researcher must be vigilant for the differences in application and able to work backwards from that point to discover which ontology modeling formula will be most effective, appropriate and less prone to error. The existing and evolving choice and advice on ontology type is considerable. As stated by Sidhu [23] when discussing classification of ontologies, differences lie in the amount of detail they express. Meersman [24] ventures three layers of knowledge: Language Knowledge, Domain Knowledge and Applications Knowledge. This description applies to the pivotal points of the PPIE ecosystem. In our model, Language Knowledge is effectively the vehicle for the journey through, to and from, Domain Knowledge; and beyond, to the Applications Knowledge servicing the user interface. The T2DM Guideline represents Upper Level ontology but is not entirely independent as it is clinically subordinate to the 'umbrella' disease guideline sphere of diabetes. Generic diabetes care ontologies emanate from health sciences, whereas the AE ontology is domain specific; but within their PPIE Applications Knowledge relationship, these become ontologically merged in a unique domain. We will also consider a new description of the knowledge layer: Interpretative Knowledge. When valid semantic understanding is heavily dependent on semantic and pragmatic discourse circumstance, as opposed to language translation alone it becomes dependent on the ability to recognize risk of ambiguity and misperception, and therefore, misunderstanding. This risk elevates with the omnipresence of professional healthcare practitioner vocabulary use in the traditional T2DM PPIE.

Accordingly this ontological construction will include relationships with discriminatory instrumentation designed to intercept problematic conversational content regardless of modality, e.g. aural or visual dialogue. Difficulties encountered with forms of questioning, and specifically with communicative dysfunction traps such as multiple choice, accentuate the need for a cultural accommodation within the ontology structure, vis-a-vis storytelling, known by Aboriginals as 'Yarning'. This is domain specific and presents our project with a significant volume collection and collation process for as yet unpublished and largely uncategorized dialectical vocabulary related to healthcare generally, and T2DM specifically.

Figure 2 presents the full healthcare ontology tree. It should be noted that the directional influence of the healthcare ontology is toward the specific chronic disease of T2DM with an intention to map to Aboriginal English. Therefore the subclass properties are clinically biased toward Aboriginal T2DM relationships.

'Person' is identified in Figure 3 as a key concept subclass. Note that we have both Family and Extended Family relationships represented. This is because there must be a distinction between blood relatives and others we name the Extended Family, whom patients in the Aboriginal communities regard, relate to and commonly refer to as their family, without doubt or equivocation. This is generally an unfamiliar orientation for western trained health practitioners. The Conditions classification relates to the fact that chronic disease, and specifically T2DM carries with it a high risk of co-morbidities, i.e. associated adverse medical conditions. 


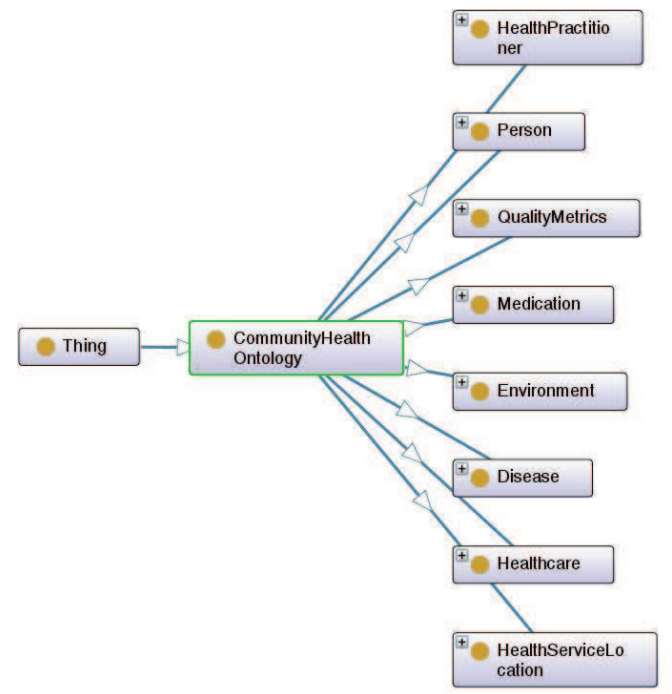

Fig. 2. Community Healthcare Ontology.

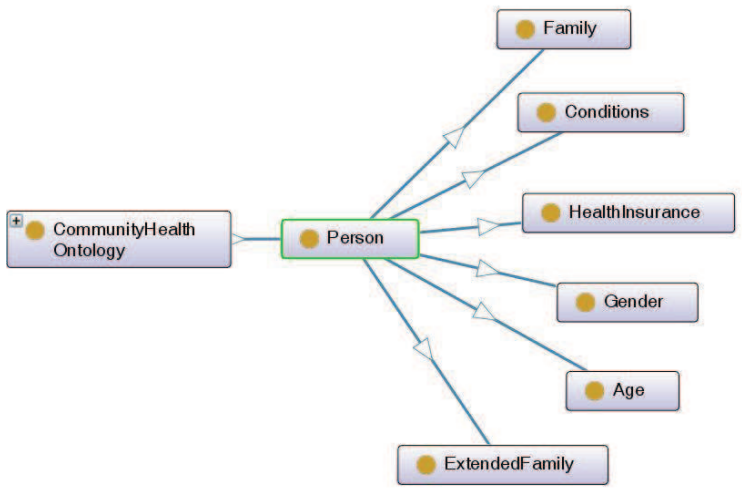

Fig. 3. Person.

The health insurance relationship is a response to the traditionally natural and commonly seasonal nomadic practices of the Aboriginal patient, recognizing that some Aboriginal patients independently traverse thousands of kilometres and do not have continuity in their healthcare engagement. The Gender classification demonstrates the socio-cultural sensitivities concerning 'men's business' and 'women's business' health status discussions in communities and clinics.

The term Health Practitioner leading the properties and relationships of Figure 4 embraces the several different professional service providers who may engage the Aboriginal patient. In the case of remote areas, and many rural clinics, General Practitioners (GPs) are infrequently present and nurses of varied description are the front line. Other Allied Health Professionals usually provide service on an occasional scheduled visit basis, often arriving and leaving the service area on the same day or within 48 hours. Figure 5 delineates the varied healthcare service locations where PPIE will be required.

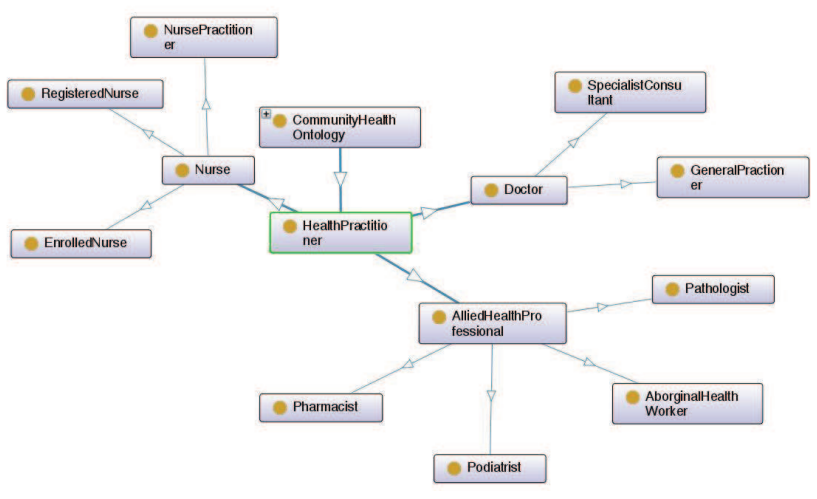

Fig. 4. Health practitioner.

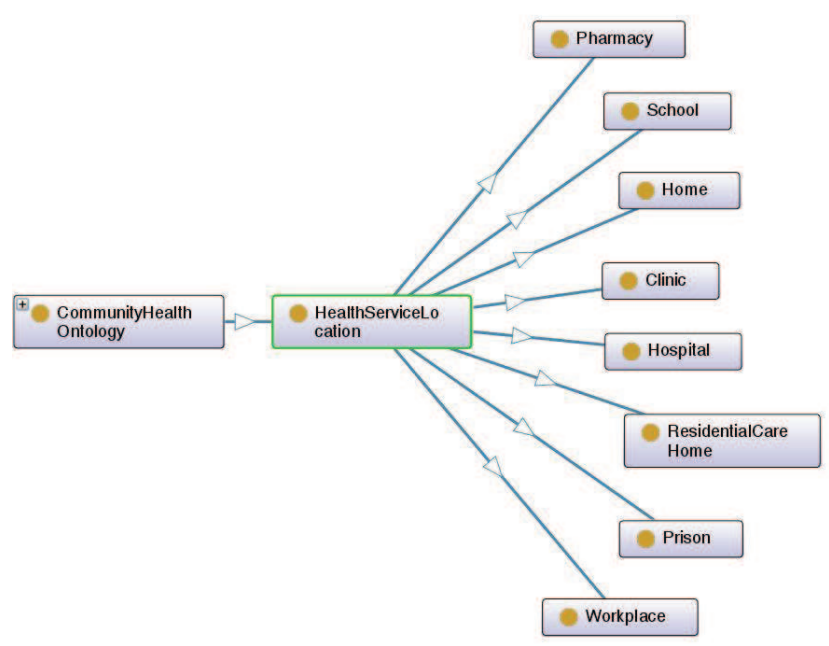

Fig. 5. Health service locations.

The environment is distinctive to location, as shown in Figure 6. The physical operating conditions, climatic factors and cultural influences help determine the special efficacy barriers to primary care interviews. Under Disease as in Figure 7, we find a simple classification set that will allow a comprehensive cover for all correlations incidental to chronic disease and T2DM specifically.

Medication as in Figure 8 is an essential part of a healthcare ontology involving the high maintenance management of T2DM. This set concisely groups the categories under which pharmaceutical product treatment plans are administered for Aboriginal patients.

Figure 9 focuses on the holistic care set that are critical process components of the T2DM PPIE treatment and care. The final subsection of the Community Healthcare 


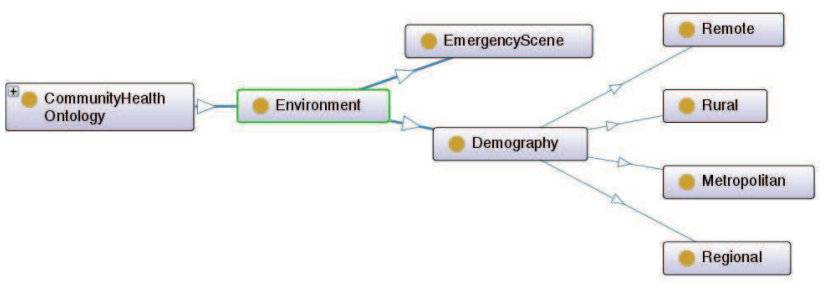

Fig. 6. Environment.

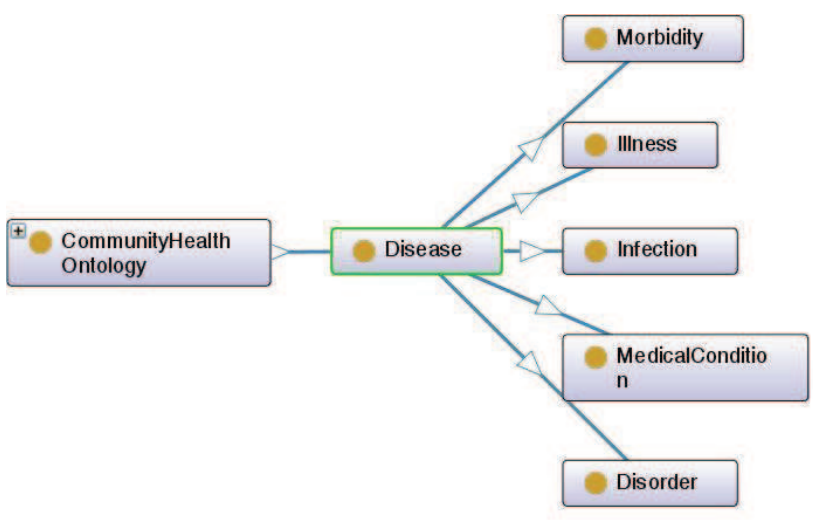

Fig. 7. Disease.

ontology as shown in Figure 10 focuses on Quality Metrics for the healthcare delivered.

Ultimately these resources will surface at the user interface, allowing patients, and practitioners, to navigate through and negotiate with, interactive communications suited to individual preferences and health literacy profiles. Touchscreen and mobile navigation buttons will enable access to and use of, conversational modalities.

Figure 11 illustrates the described composite resources and emerging ontological relationship framework.

As we begin to construct the framework for the ontological relationships, we will benefit from the contribution of Aboriginal focus groups, working together on query and response construction to strengthen the $\mathrm{AE}$ speaker contribution to PPIEs. The semantic, syntactic and pragmatic emphasis from this will take the ontology beyond the limited scope of linguistic translation of FAQs, aiming at virtually seamless interoperability between SAE and AE.

\section{Discussion}

Data collection is dedicated to evaluation of the epidemiological patterns that will determine the nature, size and eventually the geographical distribution of the $\mathrm{AE}$ speaking T2DM patient communities. The T2DM related healthcare data is indicative of the age and risk priorities for PPIE enhancements in the primary care. The estimated

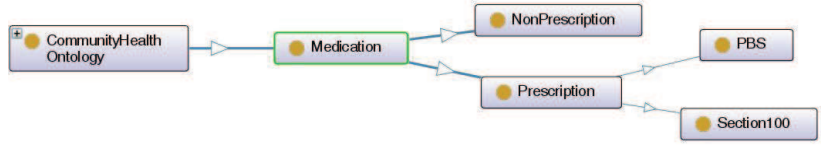

Fig. 8. Medication.

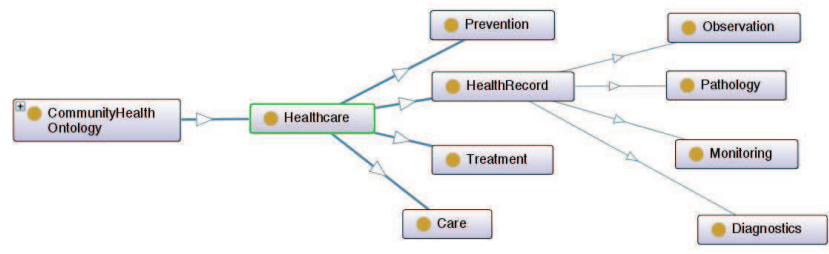

Fig. 9. Healthcare.

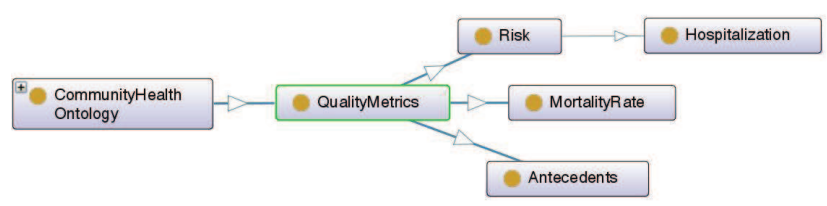

Fig. 10. Quality Metrics.

Indigenous population of Western Australia as at 30 June 2010 was 76,218 people, representing $3.4 \%$ of the WA population which itself represented an estimated $13.5 \%$ of the national population. Proportions of people reporting diabetes/high sugar levels as a long-term health condition, by Indigenous status, with Indigenous to non-Indigenous ratios, Australia, for the year 2004-2005, indicate that $1 \%$ of Indigenous people aged between 15-24 have diabetes, versus $0.5 \%$ of non-Indigenous population of the same age, a ratio of 2. In the age group 25-34 the (2004-05) percentages were $4.3 \%$ Indigenous, $0.6 \%$ non-Indigenous, a ratio of 7.2. In the age group 55 yearst the percentage of the Indigenous population with diabetes (2004-05) was 32.1\% compared with $11.2 \%$ of the non-Indigenous population, a ratio of 2.8. Overall, $2.9 \%$ of people on the National Diabetes register (NDR) in 2005-2007 were recorded as Indigenous which is slightly higher than the Indigenous population percentage in 2006 (2.6\% of the Australian population) [25]. It is to be expected that empowerment of the Aboriginal T2DM patient will result in a shift in the PPIE experience for all participants; but also that in the longer term, the educational impact will show in improved disease management and service demand outcomes. The Four Habits clinical encounter model from Frankel [8] has influenced the ontology output structure, with greater emphasis being placed on the introductory and closing phases of the PPIE, effectively resulting in six instead of four sequential components. The first of these is Patient Social Engagement (PSE); and the last is 


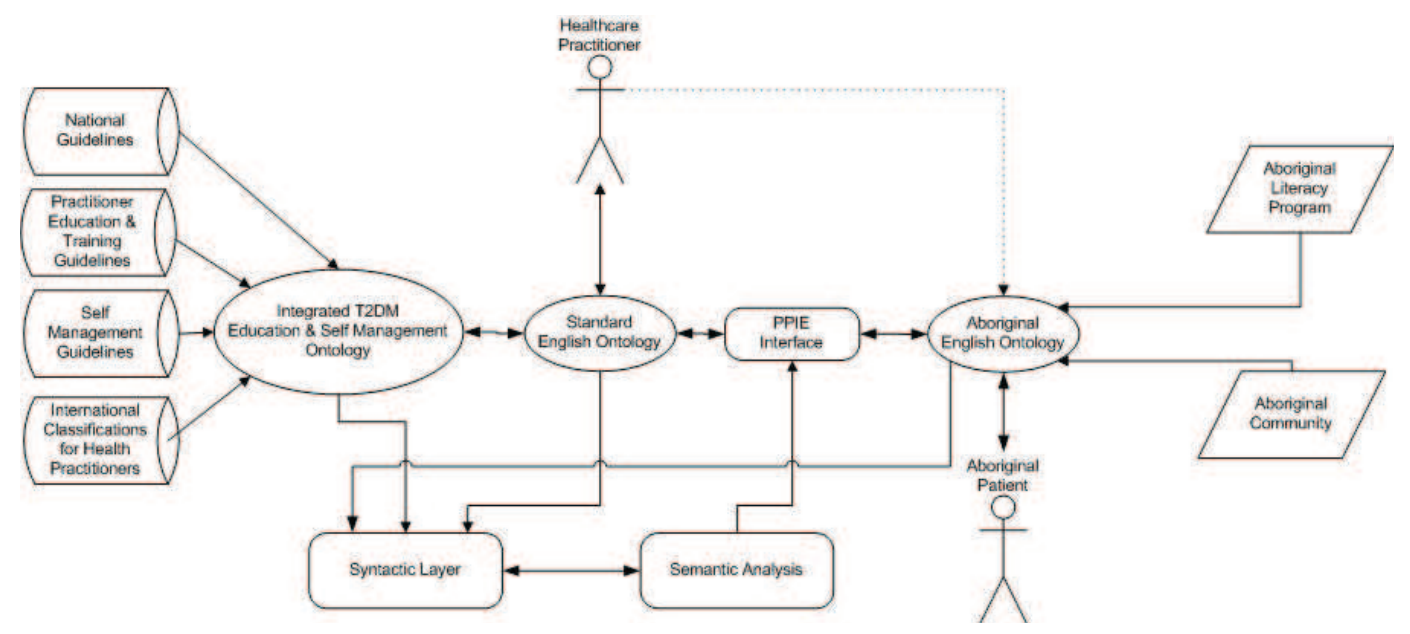

Fig. 11. PPIE ontology application framework.

the Self Management Compact (SMC). The PSE directly relates to the valuable practice commonly referred to as 'Yarning' in which Aboriginal people engagement in storytelling as a key element of an engaging, friendly (therefore non-threatening) dialogue. The SMC is investment in time and effort to encourage personal 'ownership' of T2DM, with shared responsibility between patient and practitioner to manage the disease successfully. This implicitly embraces health education aimed not only at alleviation and reduction of associated risk, but also at contributing toward T2DM prevention in the Aboriginal communities, and in particular, through patient peer support groups. In due course other ontologies will be imported in order to enrich the content within the PPIE framework, providing system flow for electronic health records and personalized care plans, both within and beyond T2DM. Chronic and associated disease prevention is priority targets and the T2DM concentration is viewed as a valid trial base for modeling.

\section{References}

[1] World Health Organization (WHO). (1968) Health promotion glossary. [Online]. Available: http://www.who.int/hpr/NPH/docs/hp_glossary_en.pdf, (Accessed: 7 July 2010)

[2] S. Ratzan and R. Parker, Health literacy, ser. National Library of Medicine current bibliographies in medicine. Bethesda, MD: National Institutes of Health, U.S. Department of Health and Human Services, 2000.

[3] S. Maggi et al., "Diabetes as a risk factor for cognitive decline in older patients," Dementia and Geriatric Cognitive Disorders, vol. 27, no. 1, 2009.

[4] E. van den Berg et al., "A 4 year follow-up study of cognitive functioning in patients with type 2 diabetes mellitus," Diabetologia, vol. 53 , no. 1 , pp. 58-65, 2010.

[5] Department of Health, Diabetes in Western Australia, Diabetes WA and Epidemiology Branch, Department of Health, Western Australia, Perth, 2009.
[6] R. M. Frankel and T. Stein, "Getting the most out of the clinical encounter: The four habits model," The Permanente Journal, vol. 3, no. 3, 1999.

[7] G.-M. Breen et al., "Improving doctorpatient communication: Examining innovative modalities vis-à-vis effective patient-centric care management technology," Journal of Medical Systems, vol. 33, no. 2, pp. 155-162, 2009.

[8] R. Trudgen, Why Warriors Lie Down and Die. Parap, NT: Aboriginal Resource and Development Services Inc., 2000.

[9] J. Nagelkerk, K. Reick, and L. Meengs, "Perceived barriers and effective strategies to diabetes self-management," Journal of Advanced Nursing, vol. 54, no. 2, pp. 151-158, 2006.

[10] B. S. Gerber et al., "Implementation and evaluation of a low-literacy diabetes education computer multimedia application," Diabetes Care, vol. 28, no. 7, pp. 1574-1580, 2006.

[11] K. Cavanaugh et al., "Addressing literacy and numeracy to improve diabetes care: two randomized controlled trials," Diabetes Care, vol. 32, no. 12, pp. 2149-2155, 2009.

[12] D. Schillinger et al., "Functional health literacy and the quality of physicianpatient communication among diabetes patients," Patient Education and Counselling, vol. 52, no. 3, pp. 315-323, 2004.

[13] L. Dyson, "Cultural issues in the adoption of information and communication technologies by indigenous australians," in Cultural Attitudes Towards Communication and Technology, Perth, Australia, 2004, pp. 58-71.

[14] L.Dyson et al., ICTS FOR INTERCULTURAL DIALOGUE An Overview of UNESCOs Indigenous Communication Project, UNESCO, N.D. [Online]. Available: http://www-staff.it.uts.edu.au/ laurel/Publications/ICTsForInterculturalDialogue.pdf

[15] F.Brady, L.Dyson, and T.Asela, "Indigenous adoption of mobile phones and oral culture," in Cultural Attitudes Towards Coтmunication and Technology, Perth, Australia, 2008, pp. 384-398.

[16] E.Hunter et al., "Pride and performance: evaluation challenges of a multimedia and information technologies project in remote aboriginal australia," Australasian Psychiatry, vol. 17, no. 1, pp. S155-S158, 2009.

[17] A. Xie et al., "Cross-cultural influence on communications effectiveness and use interface design," International Journal of Intercultural Communications, vol. 22, pp. 11-20, 2009.

[18] I. Malcolm et al., TWO-WAY English: Towards more user-friendly education for speakers of Aboriginal English. Perth, Australia: Education Dept. Western Australia, 1999.

[19] A. Cass et al., "Sharing the true stories: improving communication between aboriginal patients and healthcare workers," Medical Journal of Australia, vol. 176, no. 10, pp. 466-470., 2002.

[20] S. C. Bailin and H. B. Lehmann, "Facilitating physician-patient 
dialogue through ontology negotiation," in 16th IEEE Symposium on Computer-Based Medical Systems (CBMS), 2003, pp. 248-.

[21] D. Forbes, A. Sidhu, and J. Singh, "An aboriginal english ontology framework for patient-practitioner interview encounters," in 23rd IEEE International Symposium on Computer-Based Medical Systems (CBMS), Perth, Australia, 2010.

[22] M. Mehta and A. Corradini, "Developing a conversational agent using ontologies," in 12th international conference on Humancomputer interaction: intelligent multimodal interaction environments. Beijing, China: Springer-Verlag, 2007, pp. 154-164.

[23] A. S. Sidhu, "Current status of biomedical ontologies: Developments in 2006," in IEEE International Conference on Digital Ecosystems and Technologies (DEST), Cairns, Australia, 2007, pp. 581-585.

[24] R. Meersman, "Semantic ontology tools in is design," in 11th International Symposium on Methodologies for Intelligent Systems (ISMIS), Warsaw, Poland, 1999, pp. 30-45.

[25] N. Thomson et al., "Overview of australian indigenous health status," WA: Australian Indigenous HealthInfoNet, 2010. 